PROCEEDINGS OF THE

AMERICAN MATHEMATICAL SOCIETY

Volume 130, Number 5, Pages 1551-1555

S 0002-9939(01)06201-3

Article electronically published on October 12, 2001

\title{
NEW EXAMPLES OF NON-SLICE, ALGEBRAICALLY SLICE KNOTS
}

\author{
CHARLES LIVINGSTON
}

(Communicated by Ronald A. Fintushel)

\begin{abstract}
For $n>1$, if the Seifert form of a knotted $2 n-1$-sphere $K$ in $S^{2 n+1}$ has a metabolizer, then the knot is slice. Casson and Gordon proved that this is false in dimension three. However, in the three-dimensional case it is true that if the metabolizer has a basis represented by a strongly slice link, then $K$ is slice. The question has been asked as to whether it is sufficient that each basis element is represented by a slice knot to assure that $K$ is slice. For genus one knots this is of course true; here we present genus two counterexamples.
\end{abstract}

\section{INTRODUCTION}

Let $K$ be a knot in $S^{3}$. If $F$ is a Seifert surface for $K$ there is a Seifert form, $V_{K}$, defined on $H_{1}(F, \mathbf{Z})$. The knot $K$ is called algebraically slice if $V_{K}$ vanishes on some half-dimensional summand of $H_{1}(F, \mathbf{Z})$; such a summand is called a metabolizer for $V_{K}$. If $K$ is slice, that is, if it bounds a smooth embedded disk in $B^{4}$, then it is algebraically slice. Casson and Gordon [3, 4. proved that the converse does not hold by constructing explicit examples of algebraically slice knots that are not slice. (This was in contrast to the result proved by Levine [9, 10] that in higher dimensions the analogous condition of algebraic sliceness does imply that a knot is slice.)

If a basis for the metabolizer in $H_{1}(F, \mathbf{Z})$ is represented by a strongly slice link, one for which the components bound disjoint disks in $B^{4}$, then it is easily shown that $K$ is slice. The question has been asked whether it is sufficient to show that a basis of the metabolizer is represented by slice knots to assure that $K$ will be slice. Here we provide genus two counterexamples to show this is not the case. Litherland has previously given such an example in [11, but that paper never appeared, and the result depended on the development of a lengthy algorithm for computing Casson-Gordon invariants; that algorithm itself has not appeared in print. A recent paper by Cochran, Orr and Teichner [5] provides alternative examples to the ones presented here.

The examples here point to a much deeper question regarding classical concordance. At an empirical level, knotting in the curves representing elements in the metabolizer of the Seifert form present secondary obstructions to slicing a classical

Received by the editors August 10, 2000 and, in revised form, November 10, 2000.

1991 Mathematics Subject Classification. Primary 57M25, 57N70, 57Q60.

Key words and phrases. Knot concordance, algebraically slice.

(C)2001 American Mathematical Society 
knot. This was first made formal in Gilmer's work [7] where certain signatures of these knots were related to Casson-Gordon invariants. In the case we are considering these signatures all vanish, and hence a more subtle approach is needed. It is expected that in addition to the signatures of the individual components of the metabolizing basis there should be abelian invariants of the entire link that provide second order slicing obstructions. The examples produced here point to the existence of such obstructions, but the precise formulation is not evident and remains an open question.

The results of this paper depend only on the original definition of Casson-Gordon invariants of [3], the connected sum formula of Gilmer [7], and a simple method for computing Casson-Gordon invariants of satellite knots, first described in [12] and reformulated in 8 as needed here.

See 13 and 2 for basic results in knot theory. We will work in the smooth category, but all results extend to the topological, locally flat, category by [6].

The examples presented here were developed in response to a question of Effie Kalfagianni addressed to the author. We also wish to thank Pat Gilmer for conversations regarding this work.

\section{CASSON-GORdon InVARIANTS}

Let $K$ be a knot in $S^{3}$ with 2 -fold branched cover $M_{K}$. For a character $\chi$ : $H_{1}\left(M_{K}, \mathbf{Z}\right) \rightarrow \mathbf{Z}_{p}$, Casson and Gordon [3] define an invariant, denoted $\sigma_{1} \tau(K, \chi) \in$ Q. To simplify notation we have:

Definition 2.1. $\sigma(K, \chi)=\sigma_{1} \tau(K, \chi)$.

There is a linking form lk: $H_{1}\left(M_{K}, \mathbf{Z}\right) \times H_{1}\left(M_{K}, \mathbf{Z}\right) \rightarrow \mathbf{Q} / \mathbf{Z}$. The main result of [3] is the following.

Theorem 2.2. If $K$ is slice there is a subgroup $H \subset H_{1}\left(M_{K}, \mathbf{Z}\right)$ with $|H|^{2}=$ $\left|H_{1}\left(M_{K}, \mathbf{Z}\right)\right|$ and such that for any character $\chi$ with values in $\mathbf{Z}_{p}$ for prime $p$ that vanishes on $H, \sigma(K, \chi)=0$. Furthermore $H$ can be assumed to be a metabolizer (self-annihilating) for the linking form on $M$.

One simple result concerning the Casson-Gordon invariant is that $\sigma(K, \chi)=$ $\sigma(K,-\chi)$. A much deeper result is the additivity result proved by Gilmer [7], as we now describe. If $K=J_{1} \# J_{2}$, then $H_{1}\left(M_{K}, \mathbf{Z}\right)=H_{1}\left(M_{J_{1}}, \mathbf{Z}\right) \oplus H_{1}\left(M_{J_{2}}, \mathbf{Z}\right)$ and any character $\chi$ on $H_{1}\left(M_{K}, \mathbf{Z}\right)$ can be written as $\chi=\chi_{1} \oplus \chi_{2}$ with $\chi_{i}$ a character on $H_{1}\left(M_{J_{i}}, \mathbf{Z}\right)$.

Theorem 2.3. $\sigma(K, \chi)=\sigma\left(J_{1}, \chi_{1}\right)+\sigma\left(J_{2}, \chi_{2}\right)$.

For satellite knots there is an algorithm that simplifies the computation of its Casson-Gordon invariants. Details appear in [8, 12]; here is a summary. Suppose that $L$ is an unknotted circle in $S^{3}$ in the complement of $K$ that is null homologous in $S^{3}-K$. If a neighborhood of $L$ is removed from $S^{3}$ and replaced with the complement of a knotted circle $J$ in $S^{3}$ (with the boundaries identified so that the longitude of $L$ is identified with the meridian of $J$ and the meridian of $L$ is identified with the longitude of $J$ ), then the resulting manifold is diffeomorphic to $S^{3}$, but the curve $K$ now represents a perhaps different knot, say $K^{*}$, in $S^{3}$. (Traditionally $K^{*}$ has been called a satellite of $J$ with embellishment $K$; in effect the portion of $K$ that passes through $L$ is tied into the knot $J$.) 


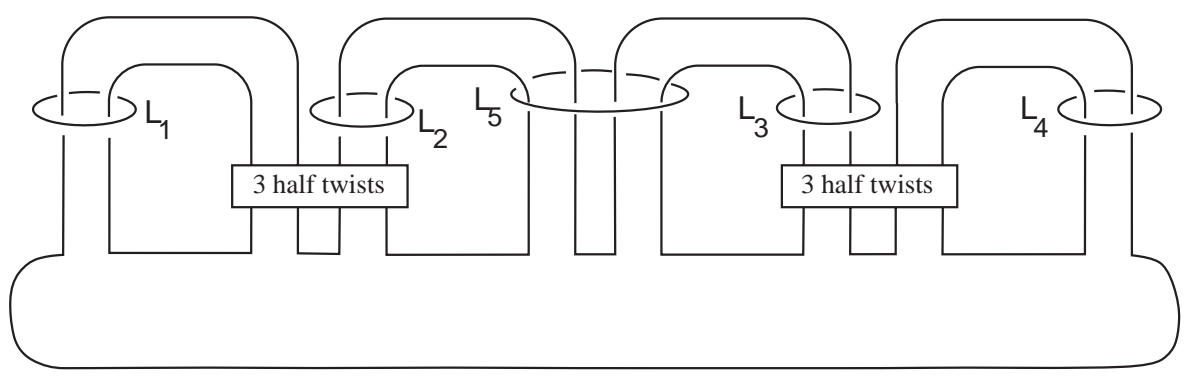

FiguRE 1.

The curve $L$ lifts to a pair of curves, $\tilde{L}^{\prime}$ and $\tilde{L}^{\prime \prime}$ in $M_{K}$. Thus, $M_{K^{*}}$ is constructed from $M_{K}$ by removing neighborhoods of $\tilde{L}^{\prime}$ and $\tilde{L}^{\prime \prime}$ and replacing both with copies of the complement of $J$. This construction leaves the homology unchanged and there is a natural correspondence between the homology, and cohomology, groups of $M_{K}$ and $M_{K^{*}}$. In particular we can identify a character $\chi$ on $H_{1}\left(M_{K^{*}}, \mathbf{Z}\right)$ with characters on $H_{1}\left(M_{K}, \mathbf{Z}\right)$. In this situation we have the following:

Theorem 2.4. $\sigma\left(K^{*}, \chi\right)=\sigma(K, \chi)+2 \sigma_{\chi\left(\tilde{L}^{\prime}\right) / p}(J)$.

Here $\sigma_{k / p}(J)$ denotes the classical Tristram-Levine signature [14] of the knot $J$, given as the signature of the hermetianized Seifert form $(1-\omega) V_{J}+(1-\bar{\omega}) V_{J}^{t}$, $\omega=e^{k 2 \pi i / p}$.

A similar result holds if $L$ is replaced with a nullhomologous unlink in the complement of $K$, with the single signature in Theorem 2.4 replaced with a sum of signatures.

\section{Genus two examples}

In this section we construct an example of a genus two knot, $K^{*}$, that is algebraically slice and for which a generating set for a metabolizer of a Seifert form can be represented by slice knots, and yet the knot itself is not slice. The construction depends on the choice of a second knot, $J$, and by using different $J$ an infinite family of such examples can be constructed.

The example is illustrated in Figure 1. In the figure, a knot $K$ is drawn along with five curves in the complement of its Seifert surface, $L_{i}, i=1, \ldots, 5$. The bands are twisted in such a way that the Seifert form for this knot is given by

$$
\left(\begin{array}{ll}
0 & 1 \\
2 & 0
\end{array}\right) \oplus\left(\begin{array}{ll}
0 & 1 \\
2 & 0
\end{array}\right)
$$

The knot $K^{*}$ is constructed by removing neighborhoods of the $L_{i}$ and replacing them with knot complements. In our case all of these will be the complement of the same knot, $J$, with the exception of $L_{5}$ which is replaced with the complement of $-J$.

Let $\left\{x_{1}, y_{1}, x_{2}, y_{2}\right\}$ denote the standard symplectic basis for the first homology of the Seifert surface for $K^{*}$ (as drawn above) and at the same time denote obvious simple curves on the Seifert surface representing these classes. (So, each curve passes over exactly one band, with $x_{1}$ going around the leftmost band.)

Notice that, as knots, $x_{1}$ and $y_{2}$ are each the knot $J$, while $y_{1}$ and $x_{2}$ are each represented by the slice knot $J \#-J$. In particular, $y_{1}$ and $x_{2}$ form a basis for 


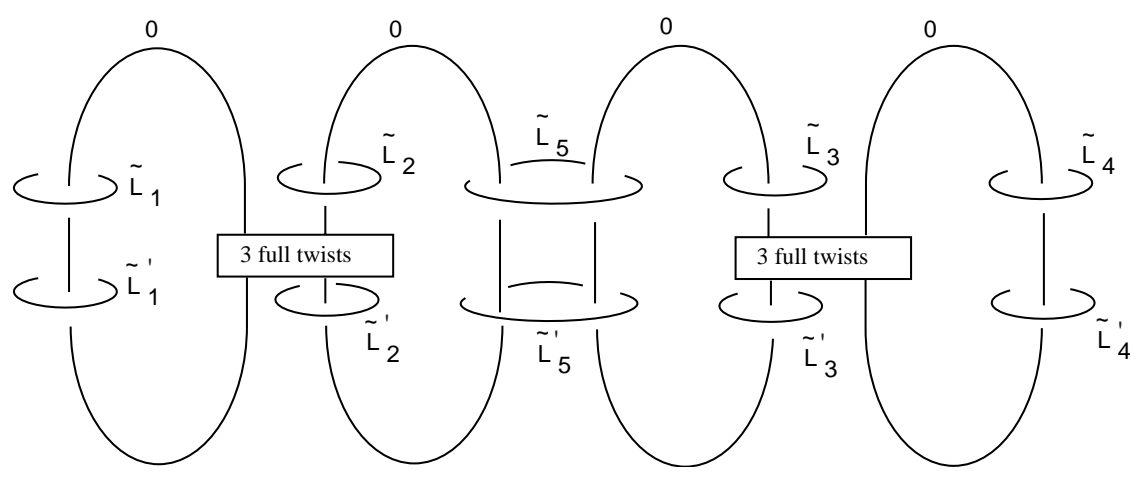

FIGURE 2.

a metabolizer for the Seifert form with both elements represented by slice knots. That this knot provides the desired examples follows from the following theorem.

Theorem 3.1. There exists a number $C>0$ such that if $\sigma_{1 / 3}(J)>C / 2$, then $K^{*}$ is not slice.

To prove this result, we begin with a diagram of the 2 -fold branched cover of $S^{3}$ branched over $K^{*}$ as drawn in Figure 2 using the algorithm of [1]. In this figure the lifts of the $L_{i}$ are drawn, and are to be replaced with the appropriate knot complement (of either $J$ or $-J$ ) to complete the construction.

The homology of the cover is $\left(\mathbf{Z}_{3}\right)^{4}$ generated by meridians to the four surgery circles. Denote by $\chi_{(a, b, c, d)}$ the $\mathbf{Z}_{3}$ valued character taking values $a, b, c$, and $d$ on the four meridians, listed as drawn from left to right, respectively. By choosing the orientations of the $\tilde{L}$ properly, we can assume that:

- $\chi_{(1,0,0,0)}$ takes value 1 on $\tilde{L_{1}}$ and value 0 on all other $\tilde{L}_{i}$.

- $\chi_{(0,1,0,0)}$ takes value 1 on $\tilde{L_{2}}$ and $\tilde{L_{5}}$ and value 0 on all other $\tilde{L_{i}}$.

- $\chi_{(0,0,1,0)}$ takes value 1 on $\tilde{L_{3}}$ and $\tilde{L_{5}}$ and value 0 on all other $\tilde{L}_{i}$.

- $\chi_{(0,0,0,1)}$ takes value 1 on $\tilde{L_{4}}$ and value 0 on all other $\tilde{L}_{i}$.

The same values are taken on the translates of the $\tilde{L}_{i}$, denoted by $\tilde{L}_{i}^{\prime}$ in the figure.

From this calculation we have the following formula:

$$
\sigma\left(K^{*}, \chi_{(a, b, c, d)}\right)=\sigma\left(K, \chi_{(a, b, c, d)}\right)+2(\bar{a}+\bar{b}+\bar{c}+\bar{d}-\overline{b+c}) \sigma_{1 / 3}(J)
$$

where $\bar{x}=1$ if $x= \pm 1 \in \mathbf{Z}_{3}$ and $\bar{x}=0$ if $x=0$.

Since the set of values of $\left\{\sigma\left(K, \chi_{(a, b, c, d)}\right)\right\}_{(a, b, c, d) \in\left(\mathbf{Z}_{3}\right)^{4}}$ represents a fixed finite set of rational numbers, it is bounded above in absolute value by some number $C$. It is clear that if we can prove that for every possible metabolizer the value of $\bar{a}+\bar{b}+\bar{c}+\bar{d}-\overline{b+c}$ is positive for some character vanishing on the metabolizer, then if $\sigma_{1 / 3}(J)>C / 2$ the corresponding Casson-Gordon invariant is nonzero.

It is easily seen that for all $b$ and $c, \bar{b}+\bar{c}-\overline{b+c} \geq 0$. Hence, $\bar{a}+\bar{b}+\bar{c}+$ $\bar{d}-\overline{b+c}$ will be positive unless both $a$ and $d$ are 0 . But if this were the case for all characters vanishing on the metabolizer, then this set of characters would necessarily be spanned by the characters $\chi_{(0,1,0,0)}$ and $\chi_{(0,0,1,0)}$. But then the set of characters would also contain $\chi_{(0,1,1,0)}$, and for this character $\bar{a}+\bar{b}+\bar{c}+\bar{d}-\overline{b+c}=1$.

We have now seen that for every metabolizer there is some character vanishing on that metabolizer for which $\bar{a}+\bar{b}+\bar{c}+\bar{d}-\overline{b+c}>0$. Since $\sigma\left(K, \chi_{(a, b, c, d)}\right) \geq-C$ 
and $\sigma_{1 / 3}(J)>C / 2$, it certainly follows that for this character $\sigma\left(K^{*}, \chi_{(a, b, c, d)}\right)>0$ and the proof is complete.

\section{REFERENCES}

1. S. Akbulut and R. Kirby, Branched covers of surfaces in 4-manifolds, Math. Ann. 252, (1979/80), 111-131. MR 82j:57001

2. G. Burde and H. Zieschang, Knots, de Gruyter Studies in Mathematics, 5, Walter de Gruyter \& Co., Berlin-New York, 1985. MR 87b:57004

3. A. Casson and C. Gordon, Cobordism of classical knots, A la recherche de la Topologie perdue, ed. by Guillou and Marin, Progress in Mathematics, Volume 62, 1986. (Originally published as Orsay Preprint, 1975.) CMP 19:16

4. A. Casson and C. Gordon, On slice knots in dimension three, Algebraic and geometric topology (Proc. Sympos. Pure Math., Stanford Univ., Stanford, Calif., 1976), Part 2, pp. 39-53 Proc. Sympos. Pure Math., XXXII, Amer. Math. Soc., Providence, R.I., 1978. MR 81g:57003

5. T. Cochran, K. Orr, and P. Teichner, Knot concordance, Whitney towers and $L^{2}$ signatures, preprint 2000, (ArXiv version at front.math.ucdavis.edu/math.GT/9908117).

6. M. Freedman and F. Quinn, Topology of 4-manifolds, Princeton Mathematical Series, 39, Princeton University Press, Princeton, NJ, 1990. MR 94b:57021

7. P. Gilmer, Slice knots in $S^{3}$, Quart. J. Math. Oxford Ser. (2) 34, (1983), no. 135, 305-322. MR 85d:57004

8. P. Gilmer and C. Livingston, The Casson-Gordon invariant and link concordance, Topology 31, (1992), no. 3, 475-492. MR 93h:57037

9. J. Levine, Knot cobordism groups in codimension two, Comment. Math. Helv. 44, (1969), 229-244. MR 39:7618

10. J. Levine, Invariants of knot cobordism, Invent. Math. 8, (1969), 98-110. MR 40:6563

11. R. Litherland, A formula for the Casson-Gordon invariant of a knot, preprint.

12. R. Litherland, Cobordism of Satellite Knots, Four-Manifold Theory, Contemporary Mathematics, eds. C. Gordon and R. Kirby, American Mathematical Society, Providence, R.I., 1984, 327-362. MR 86k:57003

13. D. Rolfsen, Knots and Links, Publish or Perish, Berkeley, CA, 1976. MR 58:24236

14. A. Tristram, Some cobordism invariants for links, Proc. Camb. Phil. Soc. 66, (1969), 251-264. MR 40:2104

Department of Mathematics, Indiana University, Bloomington, Indiana 47405

E-mail address: livingst@indiana.edu 